\title{
All-optical logic gates using a plasmonic MIM waveguide and elliptical ring resonator
}

\section{Rida El Haffar}

Abdelmalek Essaadi University: Universite Abdelmalek Essaadi

oussama mahboub ( $\nabla$ omahboub@uae.ac.ma)

National school of Applied sciences of Tetouan https://orcid.org/0000-0001-7437-2761

Abdelkrim Farkhsi

Abdelmalek Essaadi University: Universite Abdelmalek Essaadi

Mustapha Figuigue

Abdelmalek Essaadi University: Universite Abdelmalek Essaadi

\section{Research Article}

Keywords: Surface plasmons SPPs, Optical computing, Metal-Insulator-Metal (MIM) waveguides Contrast ratio, all-optical logic gates, FEM, FDT·D

Posted Date: November 9th, 2021

DOl: https://doi.org/10.21203/rs.3.rs-813665/v1

License: (1) (1) This work is licensed under a Creative Commons Attribution 4.0 International License.

Read Full License

Version of Record: A version of this preprint was published at Plasmonics on January 8th, 2022. See the published version at https://doi.org/10.1007/s11468-021-01567-7. 


\title{
All-optical logic gates using a plasmonic MIM waveguide and elliptical ring resonator
}

\author{
Rida El Haffar ${ }^{1}$. Oussama Mahboub ${ }^{2, *}$. Abdelkrim Farkhsi ${ }^{1}$. \\ Mustapha Figuigue ${ }^{2}$
}

Received: date / Accepted: date

\begin{abstract}
All-optical logic gates OR, XOR, AND and NOT based on two dimensional (2D) plasmonic metalinsulator-metal (MIM) coupled with Elliptical Ring Resonator (ERR) are presented, simulated and investigated by using the numerical method of the FEM (finite elements method). The results are compared and validated with the finite difference time domain (FDTD) method. The proposed logic gates are achieved with the same structure using the constructive and destructive optical interferences between a control signal and input signal(s). Their characterization was mainly done for two spectral regions, visible and near-infrared. A high intensity contrast ratios $(\mathrm{CR})$ between the logic states ("1" and " 0 ") can be achieved (28 dB) at these spectral regions. We introduce a new parameter, "gapthreshold ratio (GTR)", to characterize the gap between the maximum and minimum of the transmitted signal intensity for all logic gates. The suggested value of transmission threshold between logic " 0 " and logic "1" states is $T_{t h}=0.2$. A comparison of the two parameters, (CR) and (GTR), with previous works shows that the proposed structure gives very good results for all logic gates configurations. The proposed all-optical logic gates configuration can be a key components in optical processing and telecommunication devices.
\end{abstract}

1 Department of physics, Faculty of Sciences, University Abdelmalek Essaadi, Tetouan, Morocco

Tel: +212699045914

E-mail: rida.elhaffar@etu.uae.ac.ma

2 National School of Applied Sciences, University Abdelmalek Essaadi, Tetouan, Morocco

* Corresponding author: E-mail: omahboub@uae.ac.ma
Keywords Surface plasmons SPPs - Optical computing · Metal-Insulator-Metal (MIM) waveguides . Contrast ratio $\cdot$ all-optical logic gates $\cdot$ FEM $\cdot$ FDTD

\section{Introduction}

The use of all-optical devices has been subject to extensive research in recent years due to its potential applications to replace low-speed electronic components. The basic technology in an optical fiber system is subjected to "optical-electronic-optical" conversion at terminals of the emission and reception [1]. In this regard, photonics and its devices have emerged as a key technology to facilitate optic/photonic integrated circuits on-chip [2]. The challenge of the conversion "opticalelectronic-optical" can be tackled by using all-optical devices such as multiplexer/demultiplexer [3], optical filters [4], modulators and switches [5] and optical logic gates [6]. The potential of this later has encouraged researchers to explore it intensively. It uses photons as information carriers that can offer high speed, high capacity and low loss components [7].

Recently, various all-optical logic gates have been proposed in the literature. Shaik et al. proposed plasmonic logic gates based on all-optical T-shaped resonator [8], a Y-shaped resonators have been proposed in the literature by Rani et al. to realize an AND gate in photonic crystal waveguide [9], Pan et al. studied the XOR, OR and the all-optical Y-shaped NOT gate [10]. Younis et al. also reported the combination of the ring cavities and the Y-shaped line defect placed between two waveguides of the all-optical AND gate [6], Fu. et al. have also realized all-optical gate XNOR, XOR, NOT and OR by slot [11].

The surface plasmons polaritons (SPPs), electromag- 
netic waves that travel along a metal-dielectric or metalair interface, are considered as a promising alternative in high-integration optical circuits due to their ability to manipulate light at the sub-wavelength scale thus overcoming the diffraction limit [12]. They offer many opportunities for optical communication without compromising on size, power consumption and manufacturing cost $[13,14]$. Moreover, all-optical devices based on SPPs motivate many new researches to overcome the major constraints performance of the electronic devices based-on semiconductors which is the entrenched delay, high heat generation and limitations on electronic interconnections [15]. Thus, in this paper, we have used the geometry Metal-Insulator-Metal (MIM) waveguide for its inherent characteristics of subwavelength confinement [16]. Metal-Insulator-Metal (MIM) plasmonic waveguides are suitable components in signal processing, optimization, information processing and optical communication. Many configurations of MIM have been presented in the literature for designing plasmonic logic gates $[17-23]$.

In this work, we will design all-optical logic gates based on a MIM waveguides coupled to an elliptical ring resonator (ERR). There are reasonable motivations for choosing ERR resonator. First, different fabrication techniques for elliptical geometry (for example, electron beam lithography [24] or focused ion beam [25]) fabrication process may be easier and more precise than the shape with corners, such as rectangular and triangular shapes $[26,27]$. In addition, the use of sharp-angled resonators can be limited due to the high energy loss in the corners, as a result, by rounding them and choosing an optimal radius of curvature, thus, this loss of energy can be suppressed $[28,29]$. Moreover, in the elliptical shape, the various optical resonance tuning scenarios are presumably compared to the other shapes, so, the performances of the device can be adjusted by tuning the main radius and the small radius of the elliptical shape. Compared to most previous resonator systems using MIM waveguide coupled to elliptical resonators have high coupling coefficient and quality factor [30]. This structure offers a simple and efficient solution to realize all-optical logic gate, thus it is suitable for onchip optical computation and can also be used in WDM (Wavelength Division Multiplexing) systems.

In order to design all-optical logic gates, the main idea is based on the control of the constructive/destructive interference of two or more signals from light waves in two or more plasmonic waveguides. The constructive/destructive interference is considered a switching operation and devices that perform this operation called all-optical switching devices. Generally, the logic operations of plasmonic logic gates such as OR, AND, NOR,
NAND, XOR, XNOR and NOT can constitute the basic units of the future optical processor. The transmission of the waveguides may be weakened or improved due to such constructive or destructive interference with the resonant system, therefore, the output logic states "1" and "0" may be represented by the "HIGH" OR "LOW" levels of the optical power transmission at the output of the resonant system, respectively.

In this article, we propose the largest number of plasmonic logic gates with the same resonant frequency in the structure and the same plasmonic resonator, i.e., without the need to modify or reconfigure the ERR resonator used to analyze and implement the proposed plasmonic logic gates, then, we confine ourselves to study OR, XOR, AND and NOT optical logic gates. In our design, Two-dimensional (2D) metal-insulator-metal (MIM) waveguides are chosen to build the entire logic device, due to their simple configuration as well as their ability to provide light confinement at the nanoscale, low crosstalk, reasonable propagation distance making them ideal candidates for various ultra-compact devices and other characteristics [31]. By using current advanced nanolithography techniques, with which it is possible to design and implement these devices with great precision. In the next investigations, we will show that the MIM-based logic device can efficiently perform various logic functions by switching the signals in the input and in the control port without changing the phase of these signals for the same logic gate. In addition, important values of the contrast ratios between logic states "1" and "0" can be obtained. The presented device is simple, compact, efficient and can be easily integrated into other nanophotonic devices which can be operated in a wide range of frequencies, making it a promising element for optical computation and optical interconnection networks on chip and to create developments in nano-optical processors for optical computing technologies. At the end of these numerical investigations, the obtained results are compared with the values of the contrast ratio $(\mathrm{CR})$ previously reported in the literature. Our structure allows a considerable improvement of this ratio. Thus, the proposed device opens the way for the design of optical gates on a chip in the field of high-speed optical communication networks.

\section{Study of an elliptical ring resonator}

As preliminary study of optical logic gates, we propose a simple plasmonic structure made up of an input MIM waveguide, an elliptical ring resonator (ERR) and an output MIM waveguide, shown in fig.1a. In this part, our objective is to study optical properties through the 
characterization of the resonance peaks and modes behavior.

The properties of ERR are numerically investigated by using the FEM (Finite-element-method) method with COMSOL Multiphysics software and the results will be compared initially with the FDTD (Finite-difference time-domain) method by using Lumerical FDTD software. Note that all of these investigations were performed by using a two-dimensional (2D) model as the characteristics of 3D models can be approximately similar by using 2D models, provided that the thickness of the proposed structure in the third dimension is much greater than the size of the entity in the calculation plane (2D) $[32,33]$. Thus, the effect of the thickness of the substrate on the obtained results can be mathematically neglected by assuming that the structure is infinite in the third dimension [34]. In addition, the 2Dmodel gives much faster simulations for high resonance devices without compromising the accuracy of the calculation.

In the following simulations, the transmittance of the SPPs is calculated using the elements of the system diffusion matrix $T=\left|S_{21}\right|^{2}$. First, we use the finite element method (FEM), where a polarized plane wave TM (the magnetic field is perpendicular to the plane in Figure 1 (a), $(\vec{H} / / \vec{Z})$ is injected into the MIM structure [35]. The incident light wave will be coupled into the bus waveguide while the SPPs waves form up on the two metal interfaces. For the 2D-FDTD method, the simulation parameters are as follows: The grid size is set to $2 \mathrm{~nm}$ in order to keep convergence, the input source type is Gaussian modulated continuous wave field, the simulation time is 1400 fs with perfectly matched layer (PML) which is an absorbing boundary condition used to absorb the outgoing waves and to minimize simulation errors and reflections from sidewalls.

In addition, SPPs can be excited in the ERR resonator when a polarized light wave $T M$ is launched at the resonant wavelength of that resonator. For the proposed configuration, the $\mathrm{SiO}_{2}$ and silver are the dielectric and the metal considered, respectively. The silver (Ag) whose complex dielectric constant can be calculated with the Drude model:

$\epsilon_{A g}=\epsilon_{\infty}-\frac{\omega_{p}^{2}}{\omega^{2}+j \omega \gamma}$

Here, $\epsilon_{\infty}$ is the dielectric constant at the infinite frequency and $\omega_{p}$ and $\gamma$ stands for the bulk plasma frequencies and the electron collision, respectively. $\omega$ is the angular frequency of incident light. The parameters for silver can be set as $\epsilon_{\infty}=3.7, \omega_{p}=9.1 \mathrm{eV}$ and $\gamma=0.018 \mathrm{eV}$ [36]. For the $\mathrm{SiO}_{2}$, the refractive index is $n=1.474$ around the visible region wavelength

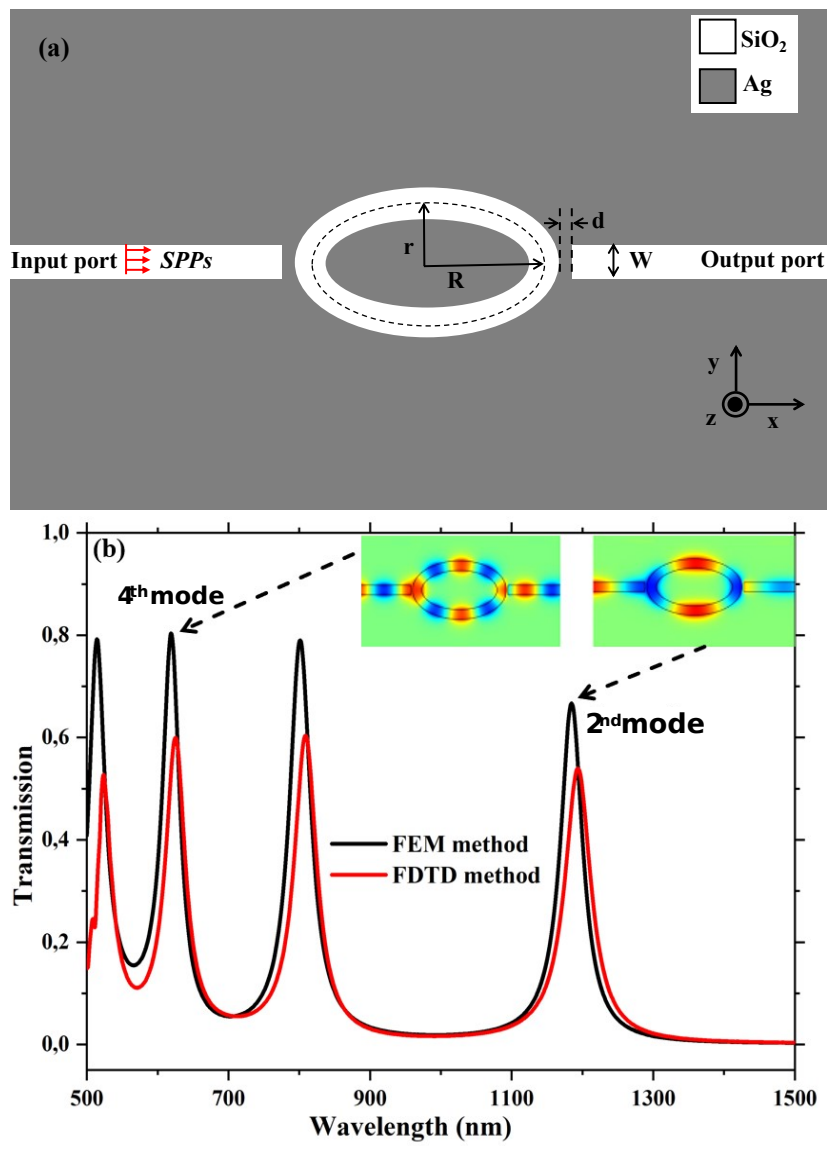

Fig. 1 a Schematic diagram of the initial ERR structure. $\mathbf{b}$ of the transmission spectrum and the magnetic field of the $2^{\text {nd }}$ and $4^{\text {th }}$ mode (The insert). The geometrical parameters of the structure are as follows: $\mathrm{w}=50 \mathrm{~nm}, \mathrm{~d}=10 \mathrm{~nm}, \mathrm{r}=125 \mathrm{~nm}$ and $\mathrm{R}=225 \mathrm{~nm}$

$\left(\lambda_{4}=684 \mathrm{~nm}\right)$ and $n=1.47$ around the near-infrared region $\left(\lambda_{2}=1186 \mathrm{~nm}\right)$ [37].

In Fig. 1(a), the geometrical parameters of the structure are: the width $(\mathrm{w})$ of the MIM waveguide, the distance (d) between the MIM waveguides and the resonator, the mean of minor radius of the elliptical ring $(\mathrm{r})$ and the mean of major radius $(\mathrm{R})$ of the elliptical ring. In this work, we use to choose the $\mathrm{w}=50 \mathrm{~nm}$ to ensure that only the fundamental transverse magnetic mode $T M_{0}$ is supported by the simulation, while the other modes are cut off.

The transmission spectrum of the ERR resonator is illustrated in Fig. 1(b) where we observe the resonance modes with transmittances of large values which are obtained at wavelengths $\lambda_{2}=1186 \mathrm{~nm}\left(T_{2}=0,67\right)$, $\lambda_{3}=803 \mathrm{~nm}\left(T_{3}=0,795\right), \lambda_{4}=624 \mathrm{~nm}\left(T_{4}=0,8\right)$ and $\lambda_{5}=514 \mathrm{~nm}\left(T_{5}=0,79\right)$. According to standing wave theory [38], the resonant wavelength of an ERR resonator is obtained as:

$\lambda \approx \frac{2 R e\left(n_{e f f}\right) L_{e f f}}{m}$ 

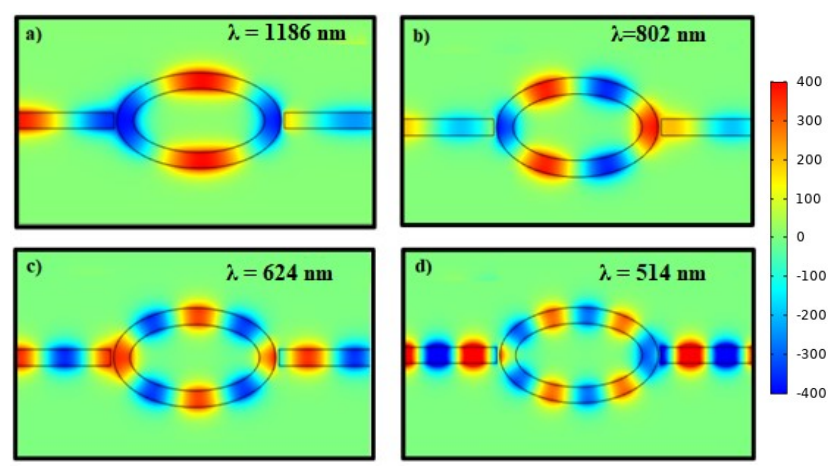

Fig. 2 a, b, c and $\mathbf{d}$ the magnetic field distributions of the ERR structure at $\lambda_{2}=1186, \lambda_{3}=802, \lambda_{4}=624$ and $\lambda_{5}=514$, respectively (Same scale)

Where $R e\left(n_{e f f}\right)$ is the real part of the effective refractive index for a wavelength in the MIM waveguide, $L_{\text {eff }}$ represents the effective length of the resonator and $\mathrm{m}$ is the order of the resonance mode ( $\mathrm{m}$ is a positive integer).

In order to consolidate the simulated results, a comparison between transmission spectrum of the MIM waveguide structure obtained with both FEM and FDTD methods is presented in Fig. 1(b). The overall shape of the both spectra is similar with a slight difference in the intensity and small shift of resonance peaks. These tiny differences are imputed to the different approaches of the two methods, such as the mesh, the boundary conditions; see reference $[27,39]$. All of the following results will be obtained by using the FEM method. In Fig. 2, we present the magnetic field distributions $|H z|$ corresponding to the resonance peaks $\left(\lambda_{2}, \lambda_{3}, \lambda_{4}, \lambda_{5}\right.$, ). We observe that $\lambda_{2}$ corresponds to $T M_{2}$ (two nodes), the $\lambda_{3}$ to $T M_{3}$ (three nodes), the $\lambda_{4}$ to $T M_{4}$ (four nodes) and the resonance $\lambda_{5}$ to $T M_{5}$ (five nodes). This observation is in good agreement with the resonance condition mentioned in analytical equation (2). For the part of the study left, a characterization of a fundamental logic functions will be presented in two regions by studying the two resonance peaks $\lambda_{2}=1186 \mathrm{~nm}$ (near-infrared) and $\lambda_{4}=624 \mathrm{~nm}$ (visible). We demonstrate the realization of the principal logic gates, i.e., OR, XOR, AND, NOT without any need of changing the structure configuration. The proposed design consists of straight waveguides and elliptical ring resonator. The width (w) of the MIM waveguide is $50 \mathrm{~nm}$, the distance (d) between the MIM waveguides and the resonator is $10 \mathrm{~nm}$, the mean of minor radius of the elliptical ring $(\mathrm{r})$ and the mean of major radius $(\mathrm{R})$ of the elliptical ring are $125 \mathrm{~nm}$ and $225 \mathrm{~nm}$, respectively. The geometrical parameters of our structure are chosen to achieve the highest power transmission ratio. A detailed study, not shown here for the sake of the purpose consistency and paper subject, was carried out.

The transmission of optical power and the position of the resonance peaks can be modified according to the geometrical parameters of the proposed structure, the selected materials, their refractive indexes, the location of the ports, the polarization of the incident field and its phase of the input light wave at the input ports. The interaction between the waveguides and the Elliptical Ring Resonator (ERR) causes new localized surface plasmon resonances, which are the result of the coupling between the resonator and the MIM waveguides. Besides, since SPPs waves only couple strongly in the near field at very short distances, the coupling distance $\mathrm{d}$ was chosen to allow this mechanism to be maintained and to obtain the best field improvement. From the results obtained by our simulations, the optimum coupling distance between the ERR and the waveguides for the initially proposed structure was chosen to be $d=10$ nm.

\section{The structure layout and characterization concept}

The proposed structure is shown schematically in fig.3(a). It consists of three straight waveguides (two input ports and one output port) and an elliptical ring resonator. The SPPs are excited by injecting a $T M_{0}$ polarized plane wave to the input ports (and the control port). The expected function for all the proposed plasmonic logic gates is achieved by the principle of constructive and destructive interferences between the input signals or between the input signals and the control signal which depends on the phase of the incident light wave. Most of the times, the performances of the four plasmonic logic gates, presented in this paper, are characterized by the following two criteria [40]: i) the transmission of the optical power ratio of input ports to the output port. This can be done by choosing a transmission threshold value in order to logically distinguish between the logical "1" value (the "HIGH" state of the transmission) and the logical "0" value ("LOW" state of the transmission) at the output. ii) the contrast ratio (CR) between the transmission of the "HIGH" and "LOW" states of the output port.

These two criteria are described by the following equations:

$T=\frac{P_{\text {in }}}{P_{\text {out }}}$

Where, $\mathrm{T}$ is the transmission, $P_{\text {out }}$ is the optical power obtained at the output port and $P_{i n}$ is the optical power injected at the input ports (and the control port). 
$C R=10 . \log \left(\frac{P_{H}^{A v r}}{P_{L}^{A v r}}\right)$

Where $P_{L}^{A v r}$ and $P_{H}^{A v r}$ are the average output powers to "0" and "1" logical states, respectively.

The Achilles heel of this later can be seen when the average output powers for the " 0 " logic state is zero or close to zero. In this case, the value of $\mathrm{CR}$ is undefined or not significant and thus, it can't be used to characterize some all-optical logic gates. Moreover, it doesn't give any indication about the gap between the maximum and minimum of the transmitted signal intensity which is essential in logical states discretization. For all these reasons, we introduce for the first time in all-optical logic gates characterization a new parameter to evaluate the optical intensity gap. We call this indicator the gap-threshold ratio (GTR).It is defined by the difference between maximum and minimum of the transmitted intensity normalized to the transmission threshold value. It can be expressed as a ratio or in $\mathrm{dB}$ :

$G T R=10 . \log \left(\frac{T_{\max }-T_{\min }}{T_{t h}}\right)$

For all-optical logic gates operations presented in this work, the transmission threshold value was chosen as $T_{t h}=0.2$.

\section{All-optical logic gates based on ERR}

\subsection{Realization of the optical OR gate}

The OR gate is designed by using the structure shown in fig. 3(a). To achieve the correct operation of OR gate, the transmission threshold value chosen is $T_{t h}=0.2$ ("1" state and " 0 " state correspond to $T>T_{t h}$ and $T<T_{t h}$, respectively). The input signals are assumed to be injected into port "A" and port "B" (fig. 3(a)). The input power $P_{i n}$ is taken $1 \mathrm{~W}$ (that corresponds to " 1 " logical state). For the near-infrared region $\left(\lambda_{2}=\right.$ $1191 \mathrm{~nm}$ ), If a single signal is injected into input A or $\mathrm{B}$, the transmission intensity at the output port is approximately $T_{2}=0.32$ which corresponds to " 1 " state according to $T_{t h}$ value. (" 0 OR $1=1$ " or " 1 OR $0=1$ " see Table. 1). For the case of the visible region $\left(\lambda_{4}=618\right.$ $\mathrm{nm})$, when the single signal is injected into input $A$ or input $\mathrm{B}$, the transmission at the output port is approximately $T_{4}=0.35$ which corresponds to " 1 " state according to the $T_{t h}$ value. When the signal is injected into both inputs simultaneously with the same phase, i.e., $\phi_{A}=\phi_{B}=0^{\circ}$; (" 1 OR $1=1$ ") the output port is close to $T_{2}=1.26$ and $T_{4}=1.43$ for the near-infrared

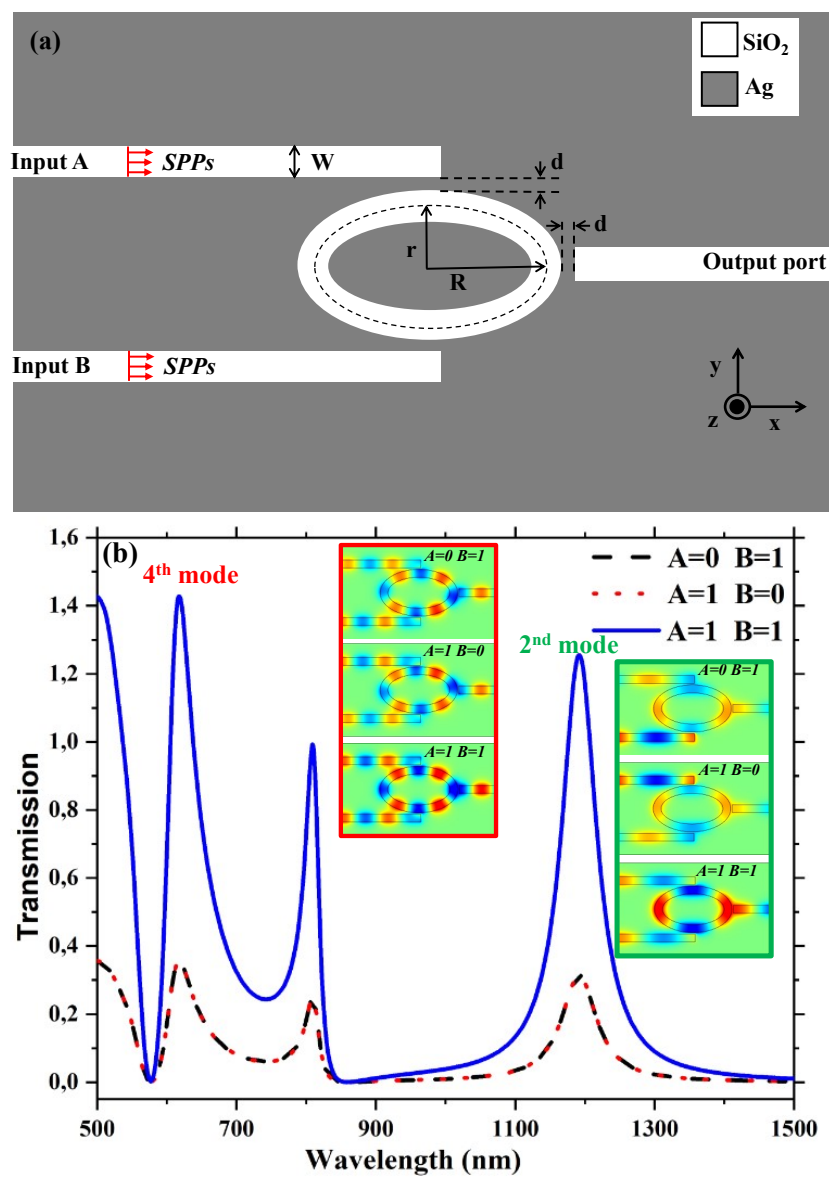

Fig. 3 a Schematic diagram of the OR gate based on an elliptical ring resonator (ERR).b The transmission spectra of the ERR resonator for different input states (Input port A and input port B) for the logic OR gate. The distributions of the magnetic fields for different combinations of the logic gate are shown in the inset of the figure (The units of the intensity profile are distributed arbitrarily between the minimum value and the maximum value). The geometrical parameters of the structure are as follows: $\mathrm{w}=50 \mathrm{~nm}, \mathrm{~d}=10 \mathrm{~nm}, \mathrm{r}=125 \mathrm{~nm}$ and $\mathrm{R}=225$ $\mathrm{nm}$

and visible region, respectively (see Table. 1). When the phase difference between the two input signals is null, a constructive interference between them occurs at the ERR and consequently leads to the high transmission intensity at output port $T>1$. In addition, the magnetic field distributions, at the inset of Fig. 3(b), of the OR gate for different input states of the two modes $\left(2^{\text {nd }}\right.$ and $4^{\text {th }}$ modes) confirm accurately this interpretation. The truth table and the transmission coefficient of the proposed OR gate are shown in Table. 1. The gap-threshold ratio for the OR gate is $G T R_{2}=2.43$ $\mathrm{dB}$ and $G T R_{4}=2.04 \mathrm{~dB}$ for the near-infrared region and the visible region, respectively. 
Table 1 The truth table and transmission spectrum for the proposed optical OR gate

\begin{tabular}{llllll}
\hline \hline Input "A" state & Input "B" state & Phase $\left(^{\circ}\right)$ & $\begin{array}{l}\text { Output } \\
\text { state }\end{array}$ & $\begin{array}{l}\text { Transmission } \\
(\lambda=1191 \mathrm{~nm})\end{array}$ & $\begin{array}{l}\text { Transmission } \\
(\lambda=618 \mathrm{~nm})\end{array}$ \\
\hline 0 & 0 & $0^{\circ}$ & 0 & 0 & 0 \\
0 & 1 & $0^{\circ}$ & 1 & 0.32 & 0.35 \\
1 & 0 & $0^{\circ}$ & 1 & $0.32\left(G T R_{2}=2.43 \mathrm{~dB}\right)$ & $0.35\left(G T R_{4}=2.04 \mathrm{~dB}\right)$ \\
1 & 1 & $0^{\circ}$ & 1 & 1.26 & 1.43 \\
\hline \hline
\end{tabular}

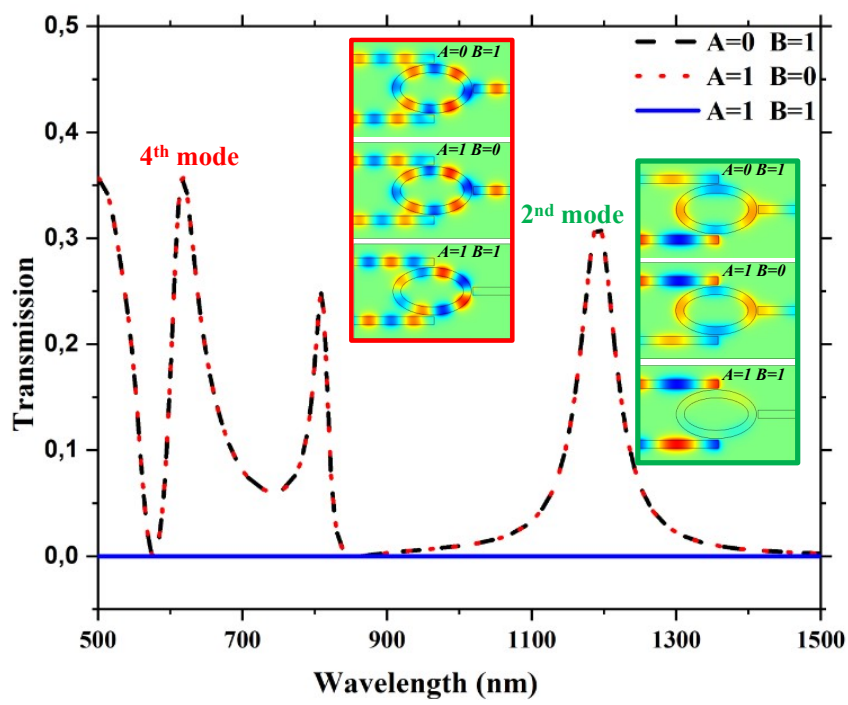

Fig. 4 The transmission of the elliptical ring resonator (ERR) for different input states (Input port A and input port B) for the logic gate XOR. The distribution of the magnetic fields for different combinations of the logic gate are shown in the inset of the figure (The units of the intensity profile are distributed arbitrarily between the minimum value and the maximum value). The geometrical parameters of the structure are as follows: $\mathrm{w}=50 \mathrm{~nm}, \mathrm{~d}=10 \mathrm{~nm}, \mathrm{r}=125 \mathrm{~nm}$ and $\mathrm{R}=225 \mathrm{~nm}$

\subsection{Realization of the optical XOR gate (EXCLUSIVE $\mathrm{OR})$}

In order to design the XOR logic gate, we have kept the same plasmonic structure at Fig. 3(a). Here, the destructive interference between the both input signals is required. Therefore, the phase difference between the two inputs $\mathrm{A}$ and $\mathrm{B}$ is set to $\Delta \phi=180^{\circ}\left(\phi_{A}=0^{\circ}\right.$ and $\left.\phi_{B}=180^{\circ}\right)$. The truth table of working principle of the gate is presented in Table. 2. When the two inputs (A and B) are at logic "1" state, the both signals are coupled to the ERR resonator and interfere destructively (due to the phase difference $\Delta \phi$ ), see the magnetic distribution field for the two modes at the insert in Fig. 4. Then, it gives rise of a weak transmitted signal at the output port (approximately zero intensity, $\left.T=7 \times 10^{-4}\right)$. For the logic operations " 0 XOR $1=1$ " or " 1 XOR $0=1$ ", the input signal is coupled to the ERR and transmitted to the output for the two resonant modes. The transmissions spectra to the output port for different input states are shown in figure 4. By using the equation (4), the contrast ratio of the XOR gate is $C R_{2}=28.75 \mathrm{~dB}$ for the $2^{\text {nd }}$ mode and $C R_{4}=28.35 \mathrm{~dB}$ for the $4^{\text {nd }}$ mode, while the gapthreshold ratio for the XOR gate is $\left(G T R_{2} \approx 2.43 \mathrm{~dB}\right)$ and $\left(G T R_{4} \approx 2.04 \mathrm{~dB}\right)$ for the near-infrared region and the visible region, respectively.

From our knowledge, these results are considered as one of the highest CR reported in the literature. The truth table and the transmission coefficient of the proposed XOR gate are shown in Table. 2.

\subsection{Realization of the optical AND gate}

To implement the optical AND logic gate, the same configuration used previously can be adopted with an additional port (probe port) as show in Fig. 5(a).

The logic function of the AND gate is performed only when both inputs are set to " 1 " logic state, the logic state " 1 " can be realized at the output port. Here, we need both constructive and destructive interference between the input signal (s) and the control signal. This later is always enabled with a phase of $\left(\phi_{P}=180^{\circ}\right.$. The AND logic operations are depending of the intensity threshold. One way to control the output signal intensity is to tune the distance $g$ between the control port and the ERR. We use to choose $\mathrm{g}=20 \mathrm{~nm}$ to properly define the intensity threshold to perform the AND output logic state (a study not shown here). When the input ports correspond to these logic operations " 1 and 0 " and "0 and 1", destructive interferences occur between input signal and control signal due to their opposite phases $\left(\phi_{A}=\phi_{B}=0^{\circ}\right.$ and control signal phase $\left.\phi_{P}=180^{\circ}\right)$, which leads to a low transmitted signal $T_{2}=0.08$ (near-infrared) and $T_{4}=0.07$ (visible). For the logic operation " 1 and 1 " a constructive interference occurs between input signals which leads to A "1" logic state with a high output intensity where $T_{2}=0.64$ (near-infrared) and $T_{4}=0.67$ (visible). The contrast ratio of the AND gate is $C R_{2}=9.5 \mathrm{~dB}$ for the $2^{\text {nd }}$ mode and $C R_{4}=9.81 \mathrm{~dB}$ for the $4^{\text {th }}$ mode, while the gapthreshold ratio for the AND gate is $G T R_{2}=4.77 \mathrm{~dB}$ and $G T R_{4}=4.47 \mathrm{~dB}$ for the near-infrared region and 
Table 2 The truth table and transmission spectrum for the proposed optical XOR gate

\begin{tabular}{llllll}
\hline \hline Input "A" state & Input "B" state & Phase $\left(^{\circ}\right)$ & Output state & Transmission $(\lambda=1191 \mathrm{~nm})$ & Transmission $(\lambda=618 \mathrm{~nm})$ \\
\hline 0 & 0 & $180^{\circ}$ & 0 & 0 & 0 \\
0 & 1 & $180^{\circ}$ & 1 & $0.32\left(C R_{2}=28.75 \mathrm{~dB}\right)$ & $0.35\left(C R_{4}=28.35 \mathrm{~dB}\right)$ \\
1 & 0 & $180^{\circ}$ & 1 & $0.32\left(G T R_{2} \approx 2.43 \mathrm{~dB}\right)$ & $0.35\left(G T R_{4} \approx 2.04 \mathrm{~dB}\right)$ \\
1 & 1 & $180^{\circ}$ & 0 & $7 \times 10^{-4}$ & $7 \times 10^{-4}$ \\
\hline \hline
\end{tabular}
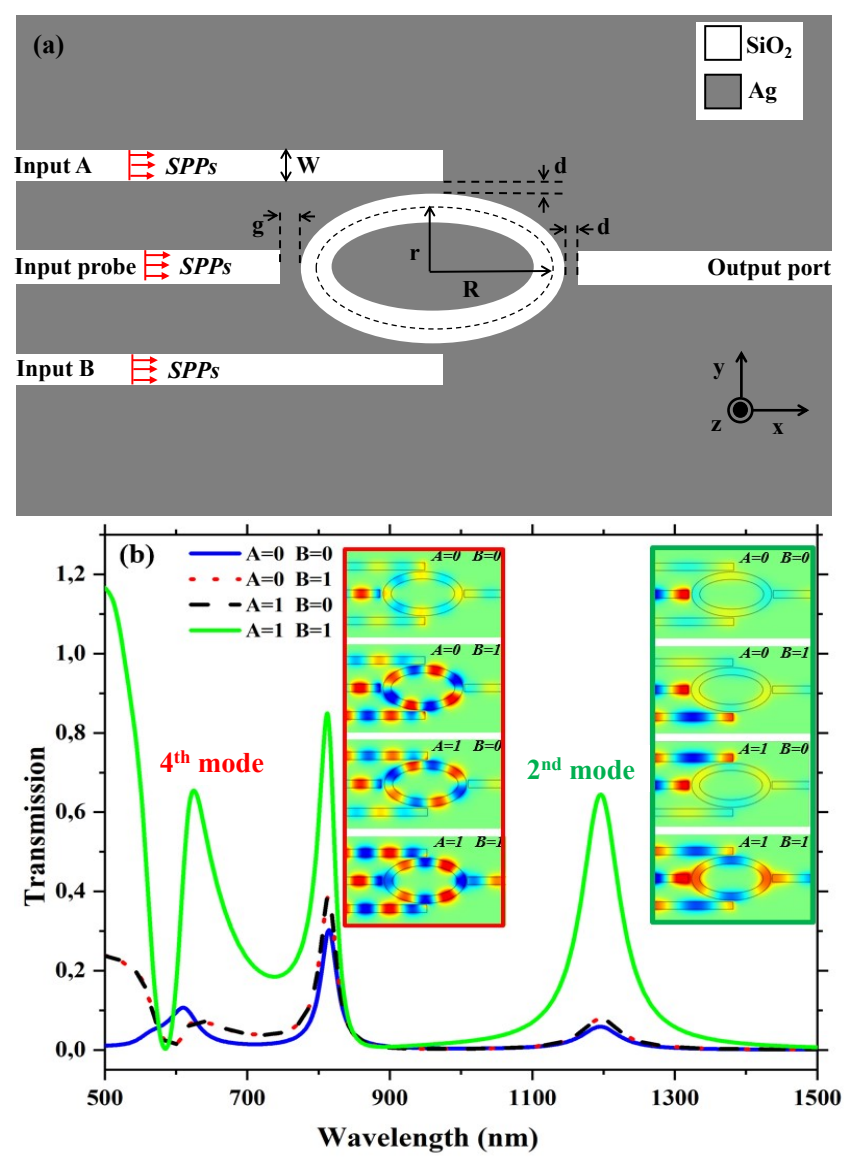

Fig. 5 a Schematic diagram of the AND logic gate based on an elliptical ring resonator (ERR). b The transmission of the resonator ERR for different input states (Input port A and input port B) for the logic AND gate. The distribution of the magnetic fields for different combinations of the logic gate are shown in the inset of the figure (The units of the intensity profile are distributed arbitrarily between the minimum value and the maximum value). The geometrical parameters of the structure are as follows: $\mathrm{w}=50 \mathrm{~nm}, \mathrm{~d}=10 \mathrm{~nm}, \mathrm{~g}=20 \mathrm{~nm}, \mathrm{r}=125 \mathrm{~nm}$ and $\mathrm{R}=225 \mathrm{~nm}$

the visible region, respectively. The truth table and the transmission coefficient of the proposed XOR gate are shown in Table. 3.

\subsection{Realization of the optical NOT gate}

A NOT gate, or inverter is used to implement the concept of complement in the switching algebra (BOOLEAN

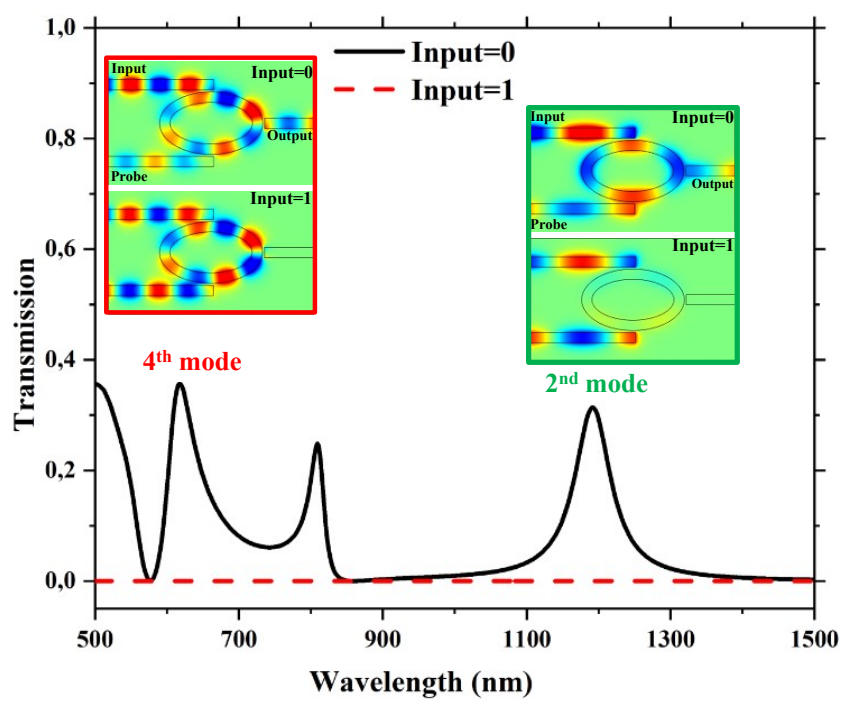

Fig. 6 The transmission of the resonator ERR for different input states (Input port) for the logic NOT gate. The distribution of the magnetic fields for different combinations of the logic gate are shown in the inset of the figure (The units of the intensity profile are distributed arbitrarily between the minimum value and the maximum value). The geometrical parameters of the structure are as follows: $\mathrm{w}=50 \mathrm{~nm}, \mathrm{~d}=10 \mathrm{~nm}, \mathrm{r}=125 \mathrm{~nm}$ and $\mathrm{R}=225 \mathrm{~nm}$.

algebra). The proposed NOT gate is similar to XOR gate where one of the two input ports becomes a control port set to " 1 " logic state, so, in order to design this logic gate, we have kept the same plasmonic structure at Fig.3(a). The function of a NOT gate can be achieved by a destructive and constructive interference between the input signal and the control signal (the inset of Fig. 6(b)). The phase difference between the input and control ports is set to $\left(\Delta \phi=180^{\circ}\left(\left(\phi_{i n}=0^{\circ}\right.\right.\right.$ et $\left(\phi_{P}=180^{\circ}\right)$. For both modes $\left(m_{2}\right.$ and $\left.m_{4}\right)$, If a single signal is injected into input port, the signal couple with the control port signal via ERR, which leads to a near-zero output. This corresponds to the logic operation "NOT $1=0$ ". On the other side, if no signal is launched into the input port, the signal of the control port couple to the ERR which leads to a high intensity signal which corresponds to the logic operation "NOT $0=1$ ". The truth table of working principle of this logic gate is presented in Table. 4. By using the equation (4), the contrast ratio of the XOR gate is $C R_{2}=28$ $\mathrm{dB}$ for the $2^{\text {nd }}$ mode and $C R_{4}=28.81 \mathrm{~dB}$ for the $4^{\text {th }}$ 
Table 3 The truth table and transmission spectrum for the proposed optical AND gate

\begin{tabular}{lllllll}
\hline \hline $\begin{array}{l}\text { Input } \\
\text { state }\end{array}$ & $\begin{array}{l}\text { Input } \\
\text { state }\end{array}$ & $\begin{array}{l}\text { Control port } \\
\text { state }\end{array}$ & Phase $\left(^{\circ}\right)$ & $\begin{array}{l}\text { Output } \\
\text { state }\end{array}$ & $\begin{array}{l}\text { Transmission }(\lambda=1191 \\
n m)\end{array}$ & $\begin{array}{l}\text { Transmission }(\lambda=618 \\
n m)\end{array}$ \\
\hline 0 & 0 & 1 & $180^{\circ}$ & 0 & 0.06 & 0.07 \\
0 & 1 & 1 & $180^{\circ}$ & 0 & $0.08\left(C R_{2}=9.5 \mathrm{~dB}\right)$ & $0.07\left(C R_{4}=9.81 \mathrm{~dB}\right)$ \\
1 & 0 & 1 & $180^{\circ}$ & 0 & $0.08\left(G T R_{2}=4.77 \mathrm{~dB}\right)$ & $0.07\left(G T R_{4}=4.47 \mathrm{~dB}\right)$ \\
1 & 1 & 1 & $180^{\circ}$ & 1 & 0.64 & 0.67 \\
\hline \hline
\end{tabular}

mode, while the gap-threshold ratio for the NOT gate is $G T R_{2}=2.9 \mathrm{~dB}$ and $G T R_{4}=2.04 \mathrm{~dB}$ for the nearinfrared region and the visible region, respectively.

\subsection{Comparison of contrast ratios for different} literatures

Table. 5 and Table. 6 summarize a comparison of the two parameters (CR) and (GTR) respectively with some previous works. The obtained (CR) is significantly high compared to other studies for XOR, AND and NOT logic gates $(28.83 \mathrm{~dB})$. As we introduce for the first time the GTR parameter, we calculated it in Table. 6 for other papers. The suggested value of transmission threshold between logic 0 and logic 1 states is $T_{t h}=0.2$ for all papers. The comparison with our GTR shows that our structure exhibits a large gap intensity between the logic states. The GTR parameter allows to compare the logic gate of the same structure between them. For our case the highest GTR is obtained for the AND logic gate $(4.77 \mathrm{~dB})$. Finally and in contrast with $\mathrm{CR}$, the GTR can be calculated for OR logic gate in our case $(2.04 \mathrm{~dB})$.

\section{Conclusion}

In this paper, a two-dimensional (2D) plasmonic metalinsulator-metal (MIM) coupled with Elliptical Ring Resonator (ERR) is studied using the numerical method of the FEM (finite elements method). The ERR configuration is proposed and characterized as an All-optical logic gates OR, XOR, AND, and NOT. The characterization was mainly done for two spectral regions, visible and near-infrared. A high intensity contrast ratios (CR) between the logic states (" 1 " and "0") was achieved (28 dB). The obtained (CR) is significantly high compared to other studies for XOR, AND and NOT logic gates $(28.83 \mathrm{~dB})$. The new parameter introduced, gapthreshold ratio (GTR), shows a good results for all logic gates compared to other studies. The GTR parameter allows to compare the logic gates of the same structure between them. For our case the highest GTR is obtained for the AND logic gate $(4.77 \mathrm{~dB})$. The proposed structure can be a good candidate as an all-optical logic gates in optical processing. 
Table 4 The truth table and transmission spectrum for the proposed optical NOT gate

\begin{tabular}{|c|c|c|c|c|c|}
\hline Input state & $\begin{array}{l}\text { Control port } \\
\text { state }\end{array}$ & Phase $\left({ }^{\circ}\right)$ & Output state & Transmission $(\lambda=1191 \mathrm{~nm})$ & Transmission $(\lambda=618 \mathrm{~nm})$ \\
\hline 0 & 1 & $180^{\circ}$ & 1 & $0.31\left(C R_{4}=28 \mathrm{~dB}\right)$ & $0.39\left(C R_{4}=28.81 \mathrm{~dB}\right)$ \\
\hline 1 & 1 & $180^{\circ}$ & 0 & $5 \times 10^{-4}\left(G T R_{2}=2.9 \mathrm{~dB}\right)$ & $5 \times 10^{-4}\left(G T R_{2}=2.04 \mathrm{~dB}\right)$ \\
\hline
\end{tabular}

Table 5 Comparison of the obtained value of the contrast ratio with different optical gates reported in the literature

CR of logic gate $(\mathrm{dB})$

\begin{tabular}{|c|c|c|c|c|c|c|c|c|c|c|}
\hline Reference & This paper & & [41] 2018 & [17] 2020 & $\begin{array}{l}{[18]} \\
2014\end{array}$ & [19] 2018 & [20] 2019 & [21] 2012 & {$[22] 2018$} & [23] 2017 \\
\hline $\begin{array}{l}\text { Numerical } \\
\text { method } \\
\text { used }\end{array}$ & 2D-FEM & & $\begin{array}{l}2 \mathrm{D}- \\
\text { FDTD }\end{array}$ & 2D-FEM & $\begin{array}{l}2 \mathrm{D}- \\
\text { FEM }\end{array}$ & $\begin{array}{l}2 \mathrm{D}- \\
\text { FDTD }\end{array}$ & $\begin{array}{l}2 \mathrm{D}- \\
\text { FDTD }\end{array}$ & $\begin{array}{l}2 \mathrm{D}- \\
\text { FDTD }\end{array}$ & 2D-FEM & $\begin{array}{l}2 \mathrm{D}- \\
\text { FDTD }\end{array}$ \\
\hline $\begin{array}{l}\text { Resonator } \\
\text { geometry }\end{array}$ & $\begin{array}{l}\text { Elliptical } \\
\text { ring } \\
\text { resonator } \\
4^{t h} \text { mode }\end{array}$ & $2^{n d}$ mode & $\begin{array}{l}2 \mathrm{D} \text { Pho- } \\
\text { tonic } \\
\text { Crystal }\end{array}$ & $\begin{array}{l}\text { Double } \\
\text { circular } \\
\text { ring res- } \\
\text { onator }\end{array}$ & $\left({ }^{(*)}\right)$ & $\begin{array}{l}\text { Circular } \\
\text { ring res- } \\
\text { onator }\end{array}$ & $\begin{array}{l}\text { octagon } \\
\text { shaped } \\
\text { ring res- } \\
\text { onator }\end{array}$ & $\begin{array}{l}\text { Circular } \\
\text { cavity } \\
\text { res- } \\
\text { onator }\end{array}$ & $\begin{array}{l}\text { Double } \\
\text { circular } \\
\text { ring res- } \\
\text { onator }\end{array}$ & $\begin{array}{l}\text { Slot } \\
\text { cavity } \\
\text { res- } \\
\text { onator }\end{array}$ \\
\hline XOR & 28.35 & 28.75 & - & - & 15 & 12.8 & 23.52 & 26 & - & 25.86 \\
\hline AND & 9.81 & 9.5 & 10.96 & - & 6 & - & - & - & - & - \\
\hline NOT & 28.83 & 28 & - & 3.16 & 15 & 18.8 & - & 26 & 11.32 & 4 \\
\hline
\end{tabular}

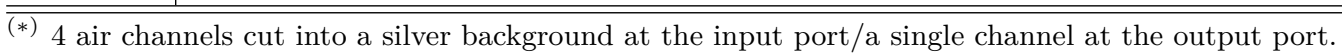

Table 6 Comparison of the obtained value of the gap-threshold ratio (dB) with different optical gates reported in the literature

Gap-threshold ratio $($ GTR $)(\mathrm{dB})$

\begin{tabular}{|c|c|c|c|c|c|}
\hline Reference & This paper & & [19] 2018 & {$[21]^{(* *)} 2012$} & {$[23]^{(* *)} 2018$} \\
\hline $\begin{array}{l}\text { Resonator } \\
\text { geometry }\end{array}$ & Elliptical ring resonator & & $\begin{array}{l}\text { Circular ring res- } \\
\text { onator }\end{array}$ & $\begin{array}{l}\text { Circular cavity res- } \\
\text { onator }\end{array}$ & $\begin{array}{l}\text { Double circular ring } \\
\text { resonator }\end{array}$ \\
\hline & $4^{t h}$ mode & $2^{n d}$ mode & & & \\
\hline OR & 2.04 & 2.43 & - & - & - \\
\hline XOR & 2.04 & 2.43 & 1.96 & 3.21 & - \\
\hline AND & 4.47 & 4.77 & - & - & - \\
\hline NOT & 2.04 & 2.9 & 3.98 & 3.21 & 4.86 \\
\hline
\end{tabular}

(**) The threshold is not defined in this paper, to calculate the GTR we use the proposed threshold in our paper $\left(T_{t h}=0.2\right)$.

Acknowledgements The authors would especially like to thank Pr. Youssef El Hafidi for the useful discussion.

Funding Not applicable.

Authors' contributions Rida El Haffar and Oussama Mahboub conceived of the presented idea. Rida El Haffar performed the computations. Mustapha Figuigue performed the comparaison with other works. Oussama Mahboub verified the analytical methods, defined the characterization concept and supervised the findings of this work. Abdelkrim Farkhsi encouraged Rida El Haffar to investigate other logic gates configurations. All authors discussed the results and contributed to the final manuscript.

Conflict of interest The authors declare that they have no conflict of interest.
Availability of data and material The authors confirm that the data supporting the findings of this study are available within the article .

Code availability Not applicable.

Ethics approval Not applicable.

Consent to participate All authors gave their consent to participate.

Consent for publication All authors gave their consent for publication.

\section{References}

1. Beheshti N, Burmeister E, Ganjali Y (2010) Optical packet buffers for backbone internet routers. IEEE/ACM Trans Networking, 18, 5, pp 1599-1609. 
2. Maier SA (2006) Plasmonics: The Promise of Highly Integrated Optical Devices. IEEE Journal of Selected Topics In Quantum Electronics, Vol. 12, No. 6, November/December(pp. 1671-1677).

3. Wang G, Lu H, Liu X, Mao D, Duan L (2011) Tunable MultiChannel Wavelength Demultiplexer based on MIM Plasmonic Nanodisk Resonators at Telecommunication Regime. OSA Publishing, Optics Express Vol. 19, No. 4 February, (Page no. 3513).

4. Zhu JH, Qi JW, Shum P, Guang Huang X (2011) A simple nanometeric plasmonic narrow-band filter structure based on metal-insulator-metal waveguide. IEEE Trans Nanotechnol 10(6):1371-1376.

5. Jonsson M (2003) Optical interconnection technology in switches, routers, and optical cross-connects. SPIE Optical Networks Magazine, 4(4):20-34,

6. Younis RM, Areed NFF, Obayya SSA (2014) Fully integrated AND and OR optical logic gates. IEEE Photon Technol Lett 26(19):1900-1903.

7. Kirchain R, Kimerling L (2007) A roadmap for nanophotonics. Nat. Photon. 1, 303-305.

8. Shaik EH, Rangaswamy N (2015) Design of photonic crystalbased all-optical AND gate using T-shaped waveguide. J. Mod. Opt. 63, 941-949.

9. Rani P, Kalra Y, Sinha RK (2013) Realization of AND gate in Y-shaped photonic crystal waveguide. Opt. Commun. 298-299, 227-231.

10. Pan D, Wei H, Xu HX (2013) Optical interferometric logic gates based on metal slot waveguide network realizing whole fundamental logic operations. Opt. Express, 21, 9556-9562.

11. Fu Y, Hu X, Lu C, Yue S, Yang H, Gong Q (2012) Alloptical logic gates based on nanoscale plasmonic slot waveguides. Nano Lett 12(11):5784-5790.

12. Chou Chau YF (2021) Multiple-Mode Bowtie Cavities for Refractive Index and Glucose Sensors Working in Visible and Near-infrared Wavelength Ranges. Plasmonics, 1557-1963.

13. Ozbay E (2006) Plasmonics: Merging photonics and electronics at nanoscale dimensions. Science 311, 189-193.

14. Anker JN, Hall WP, Lyandres O, Shah NC (2008) Biosensing with plasmonic nanosensors. Nature Mater. 7, 442-453.

15. Duan XF, Huang Y, Cui Y, Wang JF, Lieber CM (2001) Indium phosphide nanowires as building blocks for nanoscale electronic and optoelectronic devices. Nature, 409, 66-69.

16. Barnes WL, Dereux A, Ebbesen TW (2003) Surface plasmon subwavelength optics. Nature 424(6950), 824-830.

17. Fakhrulden FH, Mansour TS (2020) Design and Simulation of Plasmonic NOT Gate Based on Insulator-Metal-Insulator (IMI) waveguides. Advanced Electromagnetics, VOL. 9, NO. 1, APRIL.

18. Bian Y, Gong Q (2014) Compact all-optical interferometric logic gates based on one-dimensional metal-insulator-metal structures. Opt. Commun. 313, 27-35.

19. Zafar R, Nawaz S, Salim M (2018) Fano resonance excited all-optical XOR, XNOR, and NOT gates with high contrast ratio, Plasmonics 13, 1987-1994.

20. Moradi M, Danaie M, Orouji AA (2019) Design of alloptical XOR and XNOR logic gates based on Fano resonance in plasmonic ring resonators. Optical and Quantum Electronics, 51(5), 154.

21. Dolatabady A, Granpayeh N (2012) All optical logic gates based on two dimensional plasmonic waveguides with nanodisk resonators,J. Opt. Soc. Korea 16, 432-442.

22. Fakhrulden FH, Mansour TS (2018) All-optical NoT Gate Based on Nanoring Silver-Air Plasmonic Waveguide. International Joural of Engineering and Technology, vol. 7, pp.28182821.
23. Dolatabady A, Granpayeh N (2017) All-optical logic gates in plasmonic metal-insulator-metal nanowaveguide with slot cavity resonator. J. Nanophotonics 11(2), 026001.

24. Nagpal P, Lindquist NC, Oh SH,Norris DJ (2009) Ultrasmooth patterned metals for plasmonics and metamaterials, Science 325(5940), 594-597.

25. Vesseur EJR, Waele RD, Lezec HJ, Atwater HA, Garcia de Abajo FJ, Ploman A (2008) Surface plasmon polaritons modes in a single crystal $\mathrm{Au}$ nanoresonator fabricated using focused-ion-beam milling. Appl. Phys. Lett. 92, 0831101-083110-3.

26. Cai Y, Li Y, Nordlander P, Cremer PS (2012) Fabrication of elliptical nanorings with highly tunable and multiple plasmonic resonances, Nano Lett. 12, 4881-4888.

27. Yurkin MA (2013) Computational approaches for plasmonics, in Handbook of Molecular Plasmonics. ed. by F. Della Sala, S. D'Agostino (Pan Stanford Publishing, Singapore, pp. 83-135.

28. Panindre P, Kumar S (2016) Effect of rounding corners on optical resonances in single-mode sharp-cornered microresonators. Opt. Lett. $41,878-881$

29. Tian M, Lu P, Chen L, Lv C, Liu DM (2011) A subwavelength MIM waveguide resonator with an outer portion smooth bend structure. Opt. Commun. 284(16-17), 4078-4081.

30. El Haffar R, Farkhsi A, Mahboub O (2020) Optical properties of MIM plasmonic waveguide with an elliptical cavity resonator. Appl. Phys. A, 126, 486.

31. Veronis G, Fan SH (2005) Bends and splitters in metaldielectric-metal subwavelength plasmonic waveguides. Appl. Phys. Lett. 87(13), 131102.

32. Shibayama J, Kawai H, Yamauchi J, Nakano H, Shibayama J (2019) Analysis of a 3D MIM waveguide-based plasmonic demultiplexer using the TRC-FDTD method. Optics Communications, 452,360-365.

33. Han z, He S (2007) Two-dimensional model for threedimensional index-guided multimode plasmonic waveguides and the design of ultrasmall multimode interference split. Applied optics, Vol. 46, No. 25.

34. Xiaoyu Y, Ertian H, Mengmeng W, Yifei W, Feng W, Shubin Y (2019) Fano Resonance in a MIM Waveguide with Two Triangle Stubs Coupled with a SplitRing Nanocavity for Sensing Application. Sensors, 19(22), 4972.

35. Mahboub O, El Haffar R, Farkhsi A (2018) Optical fano resonance in MIM waveguides with a double splits ring resonator. Inernational Journal of Microwave and Optical Technology (IJMOT), vol 13, 181-187.

36. Chen Z, Yu Y, Wang Y, Guo N, Xiao L (2020) Compact Plasmonic Structure Induced Mode Excitation and Fano Resonance, Plasmonics 15, 2177-2183.

37. Gao L, Lemarchand F, Lequime M (2013) Refractive index determination of $\mathrm{SiO} 2$ layer in the $\mathrm{UV} / \mathrm{Vis} / \mathrm{NIR}$ range: spectrophotometric reverse engineering on single and bi-layer designs. J. Eur. Opt. Soc. —Rapid publications 8, 13010.

38. Su H, Yan S, Yang X, Guo J, Wang J, Hua E (2020) Sensing Features of the Fano Resonance in an MIM Waveguide Coupled with an Elliptical Ring Resonant Cavity. Appl. Sci, 10, 5096.

39. Parsons J, Burrows CP, Sambles JR, Barnes WL (2010) A comparison of techniques used to simulate the scattering of electromagnetic radiation by metallic nanostructures, J. Mod. Opt. 57(5), 356-365.

40. Abudlnabi SH, Abbas MN (2019) All-optical logic gates based on nanoring insulator- metal-insulator plasmonic waveguides at optical communications band. J. Nanophotonics Opt. 13, 016009. 
41. Subhalakshmi G, Robinson S (2018) Design and Analysis of Optical Logic Gate using Two Dimension Photonic Crystal. IEEE International Conference on Current Trends toward Converging Technologies, Coimbatore, India. 